\title{
Lehrbuch \\ der \\ Physik
}

zu eigenem Studium und zum

Gebrauche bei Vorlesungen.

\section{Dr. Eduard Riecke,}

o. Professor der Pbysik an der Universităt Gōttingen.

$\mathrm{Zwei}$ Bände.

Vierte, verbesserte und vermehrte Auflage.

Mit gegen 800 Figuren im Text.

Lex. 8. 1908. geh. $26 \mu$, geb. in Ganzleinen $28 \%$.

„... Das Buch zeigt eine Art von künstlerischem Gepräge, das die Lektüre dieses Werkes zu einem wahren Genusse macht. Ein besonders günstiger Umstand ist es, daB der Verfasser die theoretische wie die experimentelle Seite der Physik in gleichem $\mathrm{MaBe}$ beherrscht; dementsprechend sind die Beziehungen $\mathrm{zwischen}$ beiden mit einer Vollkommenheit zur Darstellung gelangt, wiesiezuvor noch nicht erreicht worden ist."

(Zeitschrift fur den physik. und chem. Unterricht.)

\section{Vorlesungen über Thermodynamik}

\section{Dr. Max Planck,}

o. ö. Professor der theoretischen Physik an der Universitat Berlin.

Mit fünf Figuren im Text.

Zweite, verbesserte Auflage.

gr. 8. 1905. kart. in Ganzleinen 7 / 50 \% .

Die fundamentalen physikalischen Eigenschaften der Krystalle

in elementarer Darstellung. Von

Dr. Woldemar Voigt,

o. Professor der Physik an der Universitat Göttingen.

Mit 52 Figuren iun Text.

8. 1898. geh. $5 \%$, geb. in Ganzleinen 5 H $60 \%$.

\section{Kompendium der theoretischen Physik. von}

Dr. Woldemar Voigt,

o. Professor der Physik an der Universităt Göttingen.

Zwei Băde.

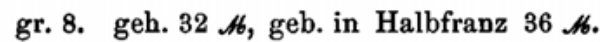

Erster Band. Mechanik starrer und nichtstarrer Körper. Wärmelehre. 1895. geh. $14 \ldots$, geb. in Halbfranz 16 .

Zweiter Band. Elektrizität und Magnetismus. Optik. 1896. geh. 18 k, geb. in Halbfranz $20 \mathrm{H}$. 


\title{
Funktionentheoretische Vorlesungen
}

\author{
Heinrich Burkhardt,
}

o. Professor der Mathematik an der Technischen Hochschule Múnchen.

$$
\text { Zwei Bände. }
$$

Mit zahlreichen Figuren im Text.

gr. 8. geh. 22 H6 60 gF, geb. in Ganzleinen 25 H 60 gF.

Ersten Bandes erstes Heft. Algebraische Analysis. Zweite, durchgesehene und vermehrte Auflage. 1908. geh. 5 \# 60 9f, geb. in Ganzleinen 6 . 60 g7.

Ersten Bandes zweites Heft. Einführung in die Theorie der analytischen Funktionen einer komplexen Veränderlichen. Dritte, durchgesehene und vermehrte Auflage.

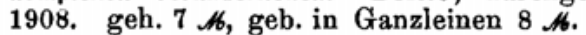

Zweiter Band. Elliptische Funktionen. Zweite, durchgesehene und verbesserte Auflage. 1906. geh. $10 \ldots$, geb. in Ganzleinen $11 \ldots$.

\section{Lehrbuch der Differentialgleichungen. Von}

\section{Dr. Heinrich Liebmann,}

Professor an der Universităt Leipzig.

Mit zahlreichen Figuren.

gr. 8. 1901. geh. $6 \mathscr{K}$, geb. in Ganzleinen 7

\section{Anwendung \\ Differential- und Integralrechnung \\ auf \\ Geometrie.}

\author{
Von \\ Dr. Georg Scheffers, \\ o. Professor an der Technischen Hochscbule Charlottenburg. \\ Zwei Bände. \\ Mit vielen Figuren im Text. \\ Lex. 8. geh. $23 \boldsymbol{H}$, geb. in Ganzleinen $25 \boldsymbol{H}$.
}

Erster Band. Einführung in die Theorie der Kurven in der Ebene und im Raume. 1901. geh. $10 \%$, geb. in Ganzleinen $11 \%$.

Zweiter Band. Einführung in die Theorie der Flächen. 1902. geh. $13 \mathscr{H}$, geb.

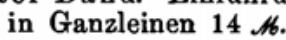




\section{Einführung}

in die

\section{Determinantentheorie}

einschließlich der unendlichen und der Fredholmschen Determinanten

von

Dr. Gerhard Kowalewski, Professor an der Universität Bonn.

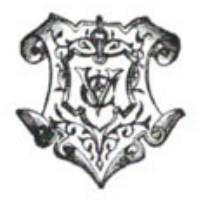

Leipzig

Verlag von Veit \& Comp.

1909 
Druck von Metzger \& Wittig in Leipzig 